\title{
SOLUTIONS FOR SAFE HOT COIL EVACUATION AND COIL HANDLING IN CASE OF THICK AND HIGH STRENGTH STEEL
}

\author{
Stefan Sieberer ${ }^{1}$, Lukas Pichler $^{1 a}$ and Manfred Hackl ${ }^{1}$ \\ ${ }^{1}$ Primetals Technologies Austria GmbH, Turmstraße 44, 4031 Linz, Austria
}

\begin{abstract}
Currently hot rolling plants are entering the market segment for thick gauges and high strength steel grades where the elastic bending property of the strip leads to internal forces in the coil during coiling operation. The strip tends to open. Primetals is investigating several possibilities to facilitate safe coil evacuation and coil handling under spring-back conditions. The contribution includes finite element models of such mechanical solutions. Results of parameter variation and stability limits of case studies are presented in the paper.
\end{abstract}

\section{Introduction}

At present, there is an increasing market demand for solutions for safe coiling and coil evacuation for hot rolling mills. This is due to the fact that currently hot rolling plants are entering the market segment for thick gauges and high strength material. In case of thick-gauge strip, the elastic bending property of the strip leads to internal forces in the coil during coiling operation. The strip tends to open. This is called 'spring-back'. Primetals is investigating several possibilities to facilitate coil evacuation and coil handling under spring-back conditions. This contribution includes finite element models to develop new mechanical solutions as well as results of parameter studies and stability investigations.

For providing a mechanism of safe evacuation and transport of spring-back coils from the downcoiler to the binding station, the so-called omega car was established. In the omega car, a circumferentially tensioned strip secures the coil during transport. In the development process of the omega car, various investigations including finite element simulations were used for development of an appropriate design concept as well as for design optimization.

Another approach to reduce the spring-back tendency is the pre-bending of the strip. Pre-bending can be achieved with the pinch roll unit in front of the downcoiler as well as with a 3-roll bending unit. Simulations were carried out with an explicit dynamic finite element solver using a 2D-model of a strip section entering the downcoiler. The results give an indication for obtainable plastic deformations with such installations.

The application of the presented numerical methods and accompanying test installations yield solutions with high potential to reduce the tendency for coils to spring-

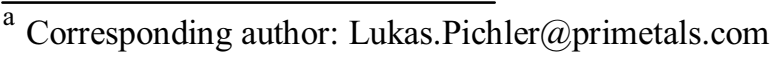

back, facilitating safe coil evacuation and coil handling operation.

\section{Mechanical issues and main influence parameters}

The main influence parameters on the coil stability are (i) the elastic bending moment resp. the stored elastic energy of the strip, (ii) the stabilizing counterforces (weight, prebending status of the strip) and the friction between the strip layers. The portion of elastic bending moment is a function of the elastic material properties, i.e. Young's modulus and moment of inertia. In the case of strip rolling this means that the thickness of the final product has the highest impact on its tendency to coil spring-back.

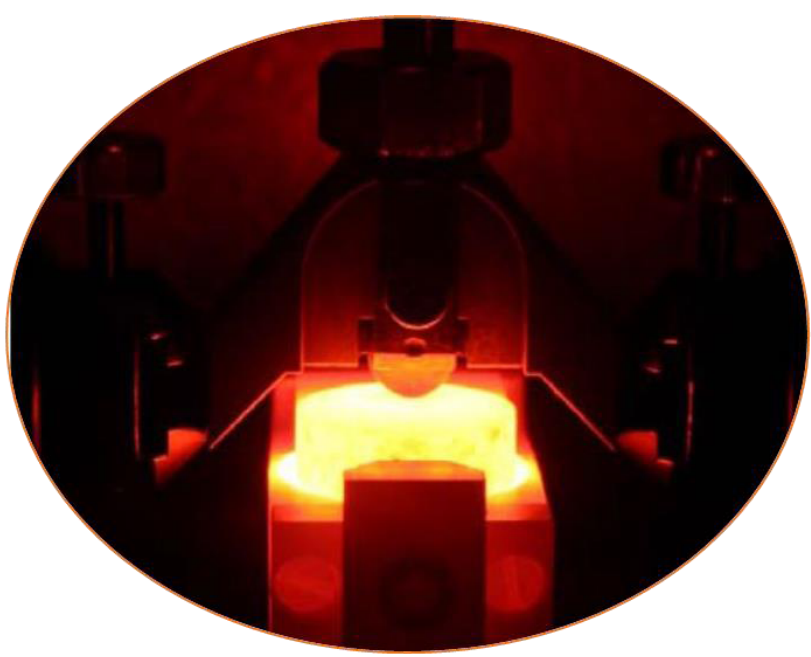

Figure 1. Tribometer - friction between cylinder and plane [1].

The stabilizing effect of the coil self-weight is limited by the maximum admissible slab length for furnace 
operation as well as the maximum admissible force acting on the coil transportation system after coiling. Thirdly, the amount of friction stabilizes the strip windings inside the coil against loosening. An experimental investigation was carried out in order to evaluate exact values for the friction coefficients (sticking and sliding friction) for the strip, depending on the current temperature range. Friction is an important parameter in hot rolling, where the coiling temperature can vary in a range of several hundred ${ }^{\circ} \mathrm{C}$. Thus, the according friction curves were evaluated for the following temperatures: $800^{\circ} \mathrm{C}, 600^{\circ} \mathrm{C}$, $400^{\circ} \mathrm{C}, 200^{\circ} \mathrm{C}$ and room temperature. The tribometer used for the experimental investigation is shown in Figure 1.

For the finite element simulation, a proper setting of friction values depending on the contact forces are obtained from the experiments (see Section 4). The contact pressure and forces were evaluated based on the experimental conditions based on the Hertz formula.

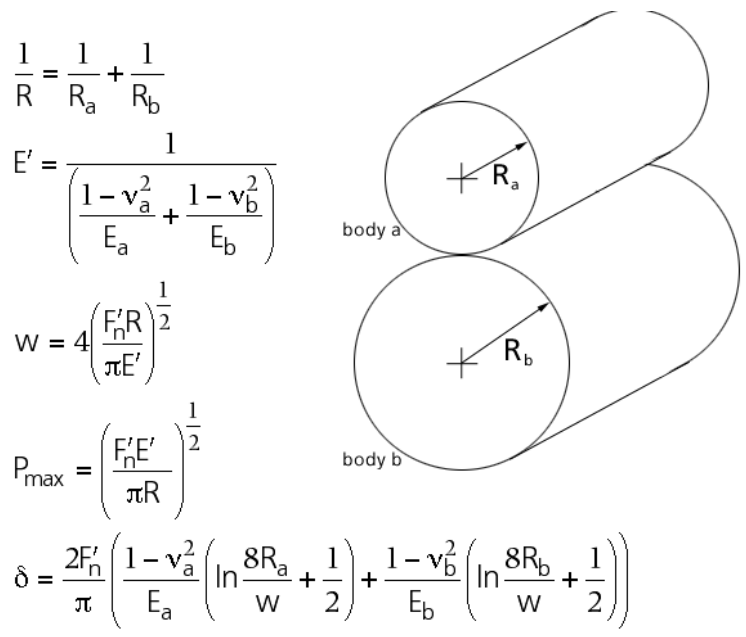

where

$\mathrm{R}_{\mathrm{a}} \ldots$ radius of cylinder, $\mathrm{R}_{\mathrm{b}} \rightarrow \infty$ (plane), $\mathrm{F}_{\mathrm{N}} \ldots$ normal force, $\mathrm{E}, v \ldots$ Young's modulus, $\mathrm{A}=1 \delta$... contact area, $\mathrm{p}=\mathrm{F}_{\mathrm{N}} / \mathrm{A} \ldots$ contact pressure, $\tau=\mathrm{F}_{\mathrm{R}} / \mathrm{A}=$ contact shear force.

\section{Pre-bending and 3-roll unit}

Figure 2 shows the model of the downcoiler assembly. The main components are (1) mandrel with core-coil, (2) pinch roll unit, (3) wrapper rolls mounted on pivot arms, (4) cradle rolls and (5) roller table. In state-of-the-art downcoilers, strip bending is performed by winding the strip around the mandrel to form the coil. The strip is tensioned by the driven mandrel and the pinch roll.

To achieve the necessary plastic strip deformation to reduce spring-back of thick-gauge steel coils, two different approaches using rollers in the downcoiler area were investigated: pre-bending of the strip (i) by lowering the roller table, and (ii) with a dedicated 3-roll unit.

For the first investigation, the roller table can be displaced vertically and a hold-down roll is placed on the strip (Figure 3). Thus, the roller table can be lowered together with the hold-down roll towards the end of the strip to create a pre-bending of the strip in the pinch-roll area. The effect on plastic strip deformation is investigated for the roller table as a standalone unit and for the assembly in the downcoiler.

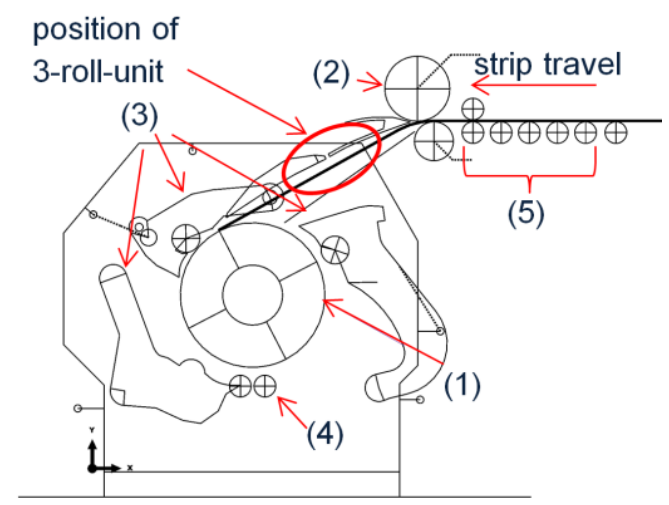

Figure 2. Model of the downcoiler assembly

For the second investigation, a 3-roll unit is modelled first to investigate the potential of the unit for induction of plastic bending of the strip (Figure 4). A 3-roll unit induces plastic deformation dependent on the relative position of the three rolls. In a second step, such a 3-roll unit is positioned in the downcoiler model and the obtained bending performance is evaluated.

\subsection{Modelling of the bending units and the downcoiler assembly}

Initially, the influence of the roller table and the three-roll unit were evaluated using 2D-Abaqus models where only the respective area was modelled. Figure 3 shows the model of pinch roll and roller table, and Figure 4 shows the model of the 3-roll unit. In these models, the strip is modelled using deformable bilinear quadrilateral elements with six elements over the strip thickness.

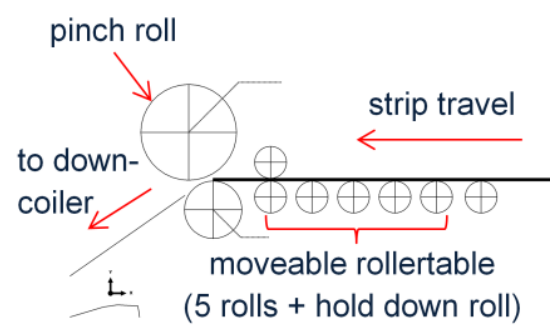

Figure 3. Model of the pinch roll unit with moveable roller table.

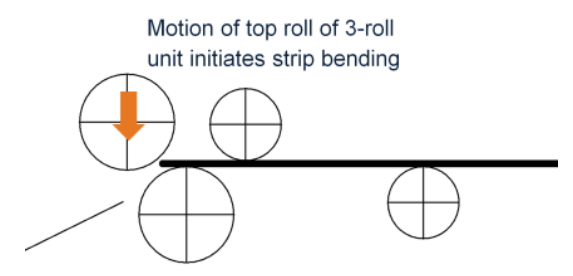

Figure 4. Model of the pinch roll unit with a moveable top roll to form a 3-roll unit. 
For simulations with the complete downcoiler, an extended 2D-Abaqus model was set-up. This model is shown in Figure 2. For performance reasons and due to the fact that only the deformation of the tail end of the strip is of interest, only the last two windings are coiled in the simulation. The mandrel with the inner windings of the coil is modelled as a solid body having equivalent mass and rotary inertia applied to it, and can be driven as in real coiling operation. Surrounding the coil are one or more wrapper rolls depending on the simulation. These wrapper rolls are standard equipment in downcoilers and facilitate the coiling of the first and last couple of windings of a coil by adjusting and retracting during the process.

The strip is fed to the coiler by means of the pinch roll unit. The pinch roll unit comprises two rollers, one top and one bottom roller of different diameter, which can be utilised to tension the strip and induce some bending of the strip. For additional guidance of the strip to the coil, two chute parts are implemented in the model.

During coiling, the coil-core is fixed in $\mathrm{x}-$ and $\mathrm{y}$ direction, and can then be released to assess the springback. At release, the coil is lowered onto the cradle rolls, and the wrapper rolls are retracted. Care is taken to apply smooth steps where possible as to minimise the potential for spring-back of the coiled strip induced by excessive force variations during solving with Abaqus/Explicit.

All modelled parts of the downcoiler are modelled using wire-features, with only the strip being modelled as a deformable 2D-structure using bilinear quadrilateral elements. To reduce hour-glassing-tendency, four elements were used over the thickness of the strip [2].

\subsection{Simulation steps}

In the initial simulations, the strip is moving through the units in one step, and the achieved bending is evaluated at a suitable point towards the end of the step.

For the extended model, the simulations are performed in a number of subsequent steps. Following the coiling and release steps is a settling step to allow the coil to fully perform eventual spring-back. The evaluation of spring back is done at the end of the settling step. To evaluate the efficiency of the different approaches to reduce spring-back, the elastic and plastic energy in the strip can be used alternatively to assessing the springback at the end of the simulation.

In all simulations, a X100 pipe-grade steel strip with thickness $25.4 \mathrm{~mm}$, yield strength $\sigma_{\mathrm{y}}=700 \mathrm{MPa}$ and friction $\mu=0.15$ (steel against steel) is used.

\subsection{Simulation results}

\subsubsection{Simulation results for roller table variation}

For the simulations including the roller table variation, the strip radius reduction is achieved by including roller table motion at the end of the strip run. Figure 5 shows plots of a simulation run. A variation of the final position of the roller table is performed, and for comparison, the radius achieved with pinch roll bending only is evaluated.

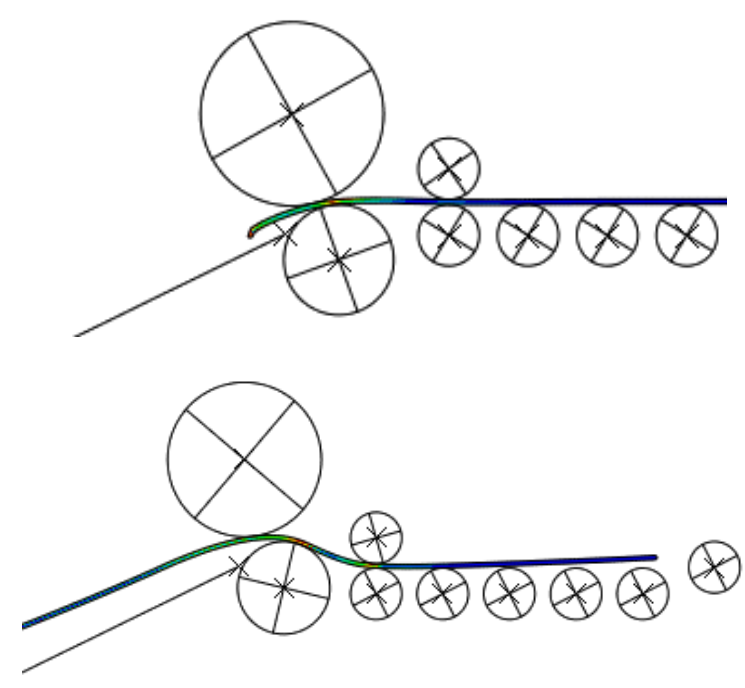

Figure 5. Simulation plots at the strip head (top) and strip tail (bottom) with the roller table lowered towards the end of the strip.

Results for the achieved bending radii with the lowered roller table are shown in Table 1. The large effect of some variation of the roller table position is not increased when the lowering is excessive, leading to sticking of the strip in the pinch roll under the assumed operational tension applied to the strip in coiling.

Table 1. Results for the roller table simulations.

\begin{tabular}{|c|c|c|c|c|}
\hline simulation & 1 & 2 & 3 & 4 \\
\hline $\begin{array}{c}\text { roller table } \\
\text { lowered (mm) }\end{array}$ & 0 & 100 & 150 & 200 \\
\hline $\begin{array}{c}\text { bending } \\
\text { radius (m) }\end{array}$ & 4.82 & 1.92 & 1.81 & sticking \\
\hline
\end{tabular}

\subsubsection{Simulation results for the 3-roll unit}

Figure 6 shows the 3-roll unit, which was modelled in the place of the top pinch-roll and the strip motion was reduced to an initial velocity in the 3-roll unit simulations. The achieved radii for a variation of the upper roll motion are shown in Figure 7. For the considered range of values, a linear relationship of roll motion to bending radius can be found.

The results show the effectivity of the 3-roll unit in strip bending. However, the simulations with the 3-roll unit do not include contact with the chute as no such contact would occur when placed in the chute area close to the coil. To evaluate the performance of both, the lowered roller table and the 3-roll unit in a downcoiler assembly, simulations were performed which are shown in the next subsection. 

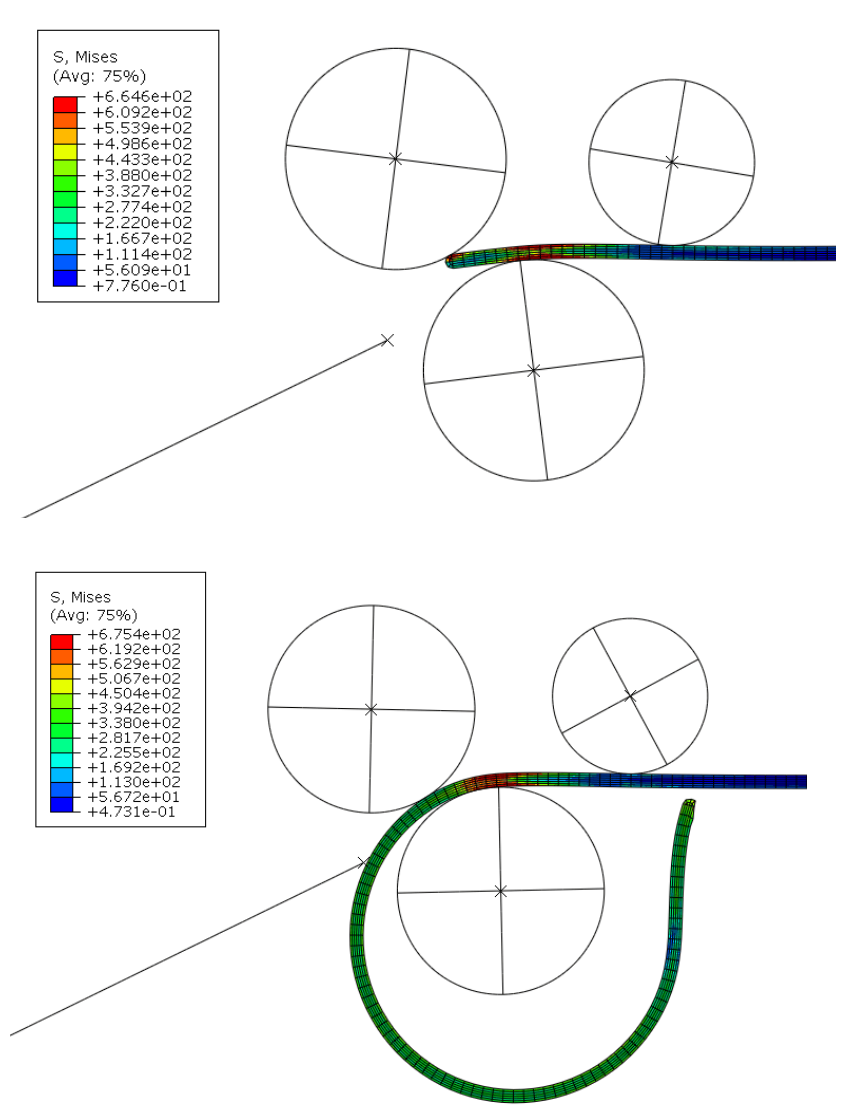

Figure 6. Simulation plots of the pinch roll used as 3-roll unit for pre-bending. Strip entering the unit (top) and strip with plastic bending (bottom).

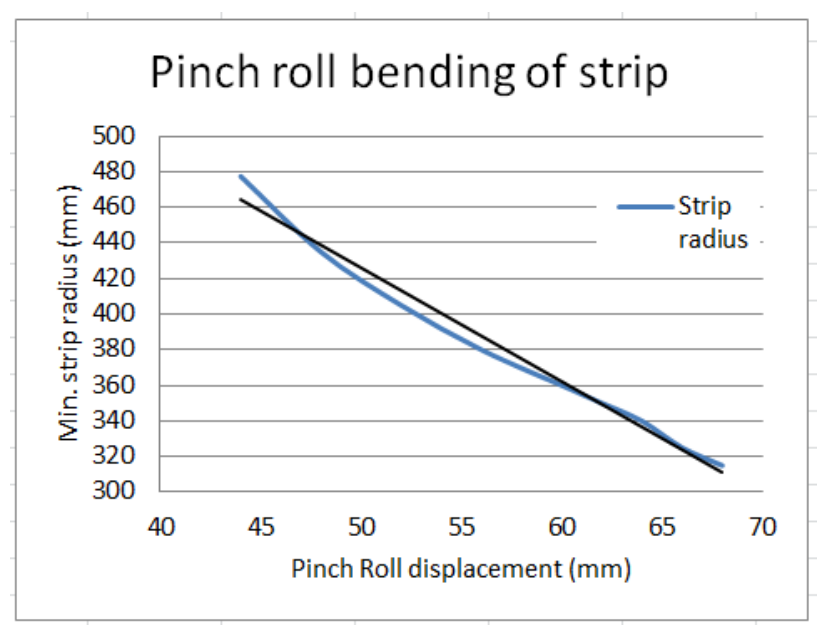

Figure 7. Resulting obtained minimum strip radius as a function of the motion of the moveable roll of the 3-roll unit (blue). The linear fit curve is shown in black.

\subsubsection{Simulations of the downcoiler assembly}

For simulations with the downcoiler assembly, (i) the movable roller table was included or (ii) a 3-roll unit was placed in the chute area (see highlighted region in Figure 2). Simulations of both arrangements were performed and the resulting outer coil diameters evaluated. The coil shape and outer diameter is an easy measure of springback.
Also, the amount of elastic energy stored in the system and the plastic energy applied to the strip is evaluated at the end of the coiling. However, it is difficult to yield a reliable spring-back measure from an elastic energy measure for reasons of complicated frictionrelated interaction of the strip windings. Also, the amount of plastic deformation gives a measure of the overall plastic deformation applied to the strip, including bending against the direction of coiling (e.g. in the 3-roll unit).

The result of a simulation with no pre-bending is shown in Figures 8 and 9. The resulting elastic and plastic energies the strip and the coil diameter at the end of the simulations are summarised in Table 2 as normalised values.

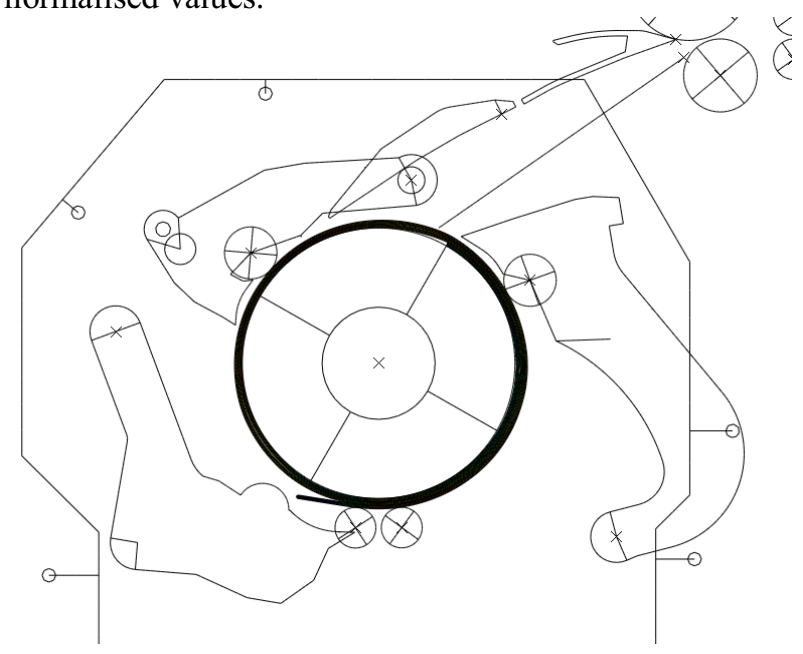

Figure 8. Simulation result of the coil with no pre-bending. Coiling is finished and the wrapper rolls are still adjusted.

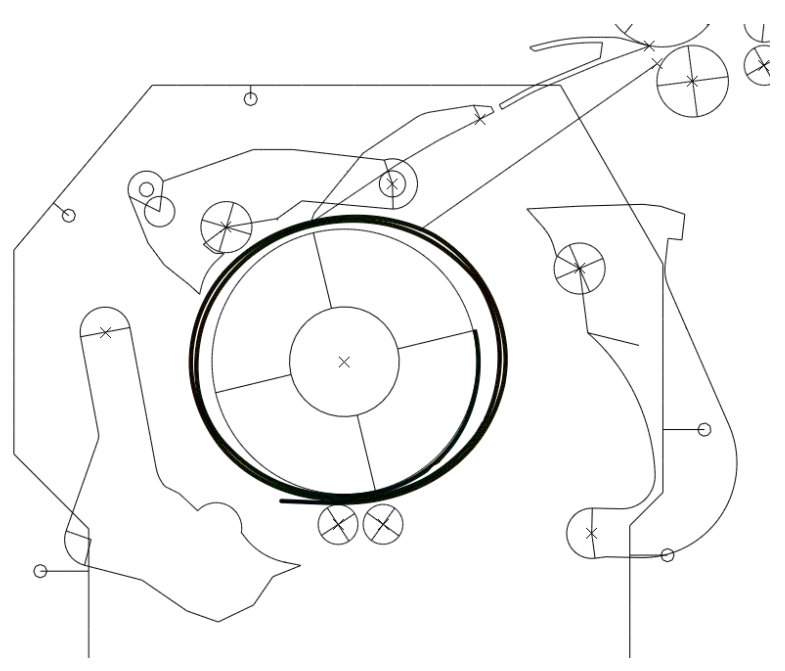

Figure 9. Simulation result of the coil with no pre-bending. The pronounced spring-back is visible at the end of the coiling with the wrapper rolls retracted.

The relative spring-back, which is defined as the maximum coil diameter compared to the diameter obtained with no spring-back, has been reduced by either of the two pre-bending measures by $34 \%$. The strain energy in the coil compared to the simulation with no pre-bending is slightly less for the version with lowered 
roller table, whereas more strain energy is retained in the case of the 3-roll unit.

Table 2. Results of the process simulations.

\begin{tabular}{|c|c|c|c|}
\hline & $\begin{array}{c}\text { rel. spring- } \\
\text { back }\end{array}$ & $\begin{array}{c}\text { rel. strain } \\
\text { energy }\end{array}$ & $\begin{array}{c}\text { rel. plastic } \\
\text { def. energy }\end{array}$ \\
\hline $\begin{array}{c}\text { No pre- } \\
\text { bending }\end{array}$ & 1 & 1 & 1 \\
\hline $\begin{array}{c}\text { lower roller } \\
\text { table }\end{array}$ & 0.66 & 0.97 & 1.91 \\
\hline 3-roll unit & 0.66 & 1.11 & 7.60 \\
\hline
\end{tabular}

The relative plastic deformation is significantly higher in both cases, as the lowered roller table as well as the 3roll unit induce plastic deformation in the strip.

\section{Coiling simulation considering friction according to experimental results}

Two different friction models are used in order to simulate the coiling of hot strip, with the goal to reproduce the real hot rolling conditions as close as possible: (i) consideration of static friction only between the windings $-\mu=0.15$ for steel against steel and (ii) an advanced friction model considering the friction coefficient, the slip rate and the contact pressure according to experimental data. The numerical investigation is carried out for two different pipe grade materials. The first material corresponds to X70 $\left(\sigma_{\mathrm{y}}=490\right.$ $\mathrm{MPa})$, the second to $\mathrm{X} 100\left(\sigma_{\mathrm{y}}=700 \mathrm{MPa}\right)$. The strip thickness is assumed to be $12 \mathrm{~mm}$, the total coil weight to be 35 tons.

A comparison is carried out first for material 1 (steel grade X70). The used factor $\mu=0.15$ steel-steel for contact provides conservative results (see Figure 10). The coil is not stable after retraction of the wrapper rolls from the coil and springs open. When the advanced friction model is applied and compared to industrial data; this results in stable conditions which are in good agreement with the observed real life situation (see Figure 11). Thus, the advanced friction model is used for assessment of coil stability. The according material and friction data is shown in Tables 3 and 4.

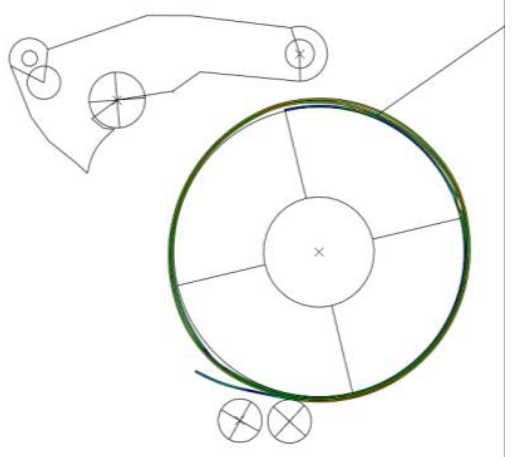

Figure 10. Friction model (i) sticking friction $\mu=0.15$; the coil falls off the support rolls.

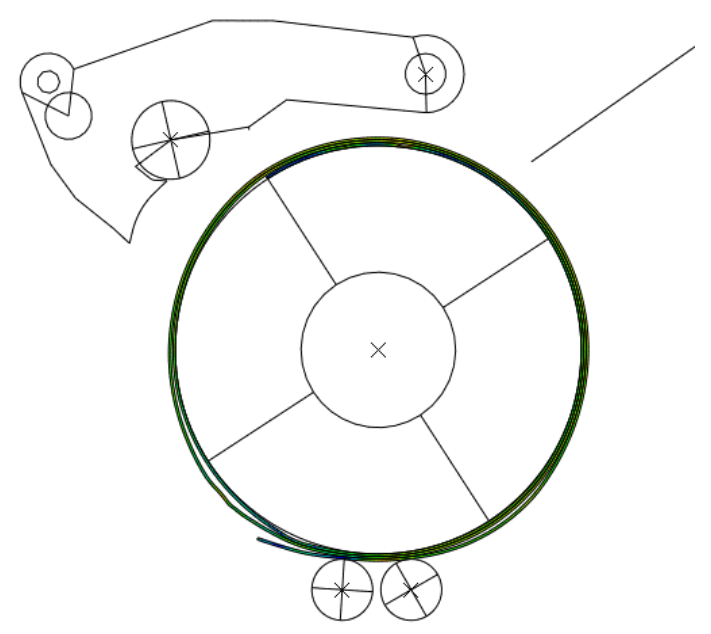

Figure 11. Advanced friction model (ii) combined friction model (sticking and gliding); the coil stays on the support rolls.

Table 3. Sticking friction

\begin{tabular}{|c|c|c|}
\hline & $\begin{array}{c}\text { Normal force } \\
30 \mathrm{kN}\end{array}$ & $\begin{array}{c}\text { Normal force } \\
80 \mathrm{kN}\end{array}$ \\
\hline Material 1 (X70) & $\mu=3$ & $\mu=1.5$ \\
\hline Material 2 (X100) & $\mu=2$ & $\mu=1.2$ \\
\hline
\end{tabular}

A contact pressure of $68 \mathrm{MPa}$ is obtained from a normal force of $30 \mathrm{kN}$ and a contact pressure of $110 \mathrm{MPa}$ from $80 \mathrm{kN}$ according to evaluation of the measurement data. The normal force acting on the coil windings increases with the coil diameter and the associated increasing coil weight during the coiling process.

Table 4. Friction model.

\begin{tabular}{|c|c|c|c|}
\hline & $\begin{array}{c}\text { Friction } \\
\text { coefficient }\end{array}$ & Slip rate & $\begin{array}{c}\text { Contact } \\
\text { pressure } \\
(\mathrm{MPa})\end{array}$ \\
\hline $\begin{array}{c}\text { Material 1 } \\
(\mathrm{X} 70)\end{array}$ & 3 & 0 & 68 \\
\hline & 0.15 & 1 & 68 \\
\hline & 1.5 & 0 & 110 \\
\hline & 0.15 & 1 & 110 \\
\hline
\end{tabular}

The conclusion of the numerical investigation is that consideration of the detailed information from the experimental friction tests leads to results which are close to reality. Consideration of sticking friction of $\mu=0.15$ only leads to a coil which drops from the support roll unit. Consideration of the experimental friction data delivers a stable coil which is in correspondence to the situations on-site a hot strip mill. Coils of material X70 and a thickness of $12 \mathrm{~mm}$ do not fall off the cradle rolls in daily operation. 


\section{Concept for safe coil evacuation and safe coil handling}

A mechanical equipment was recently developed, which provides a viable approach in order to assure safe evacuation of the coil as well as safe transport to the binding machine. The so-called "omega-car" (see also Ref. [3] for the according patent application) as shown in Figure 12 is an appropriate device to solve the abovementioned requirements. A securing strap is guided inside a U-profile. The role of the securing strap is to tighten and thus secure the coil. The entire omega shaped frame is mounted on guiding rails and is moved into the downcoiler by means of a winch drive.

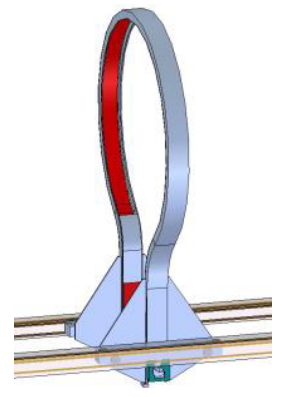

Figure 12. Omega car - frame

Figure 13 shows the omega car, which has already entered the downcoiler and is ready to tighten the finally coiled hot strip.

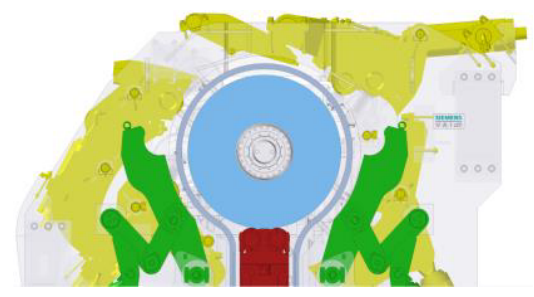

Figure 13. Omega car inside downcoiler - front view

The secured coil is transported to the binding station (see Figure 14). Inside the downcoiler, the omega frame is docked to the coil stripper car (shown in red), which acts from this step on as the master equipment to further transport the coil. Clock-springing is avoided due to the fact that a strap secures the outer windings of the coil.

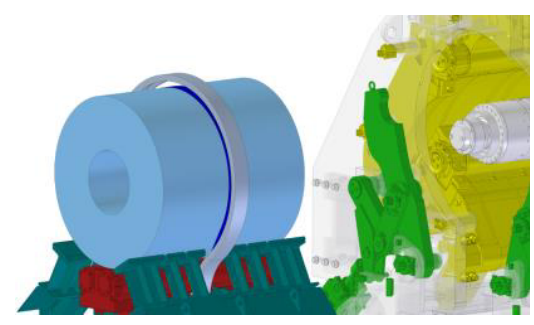

Figure 14. Omega car at binding station $-3 d$ view

As a first step to realise this concept for practical use, the basic design for a test rig installation has been carried out (see Figure 15).

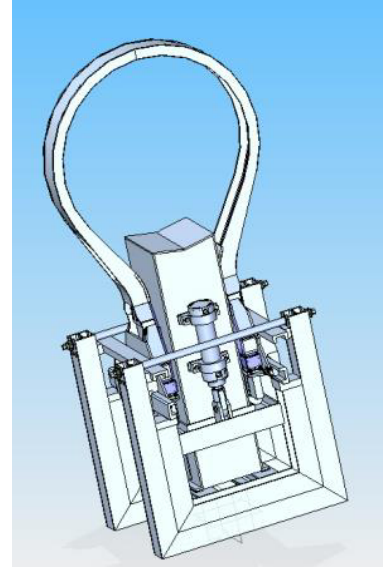

Figure 15. Basic design of test rig installation

Checks on structural integrity of the frame as well as the stability of banding strip were carried out. Also, the deformations of the rollers which connect the frame with the rail system were considered for the analysis. The forces acting on the frame and the support structure as well are shown in Figure 16.

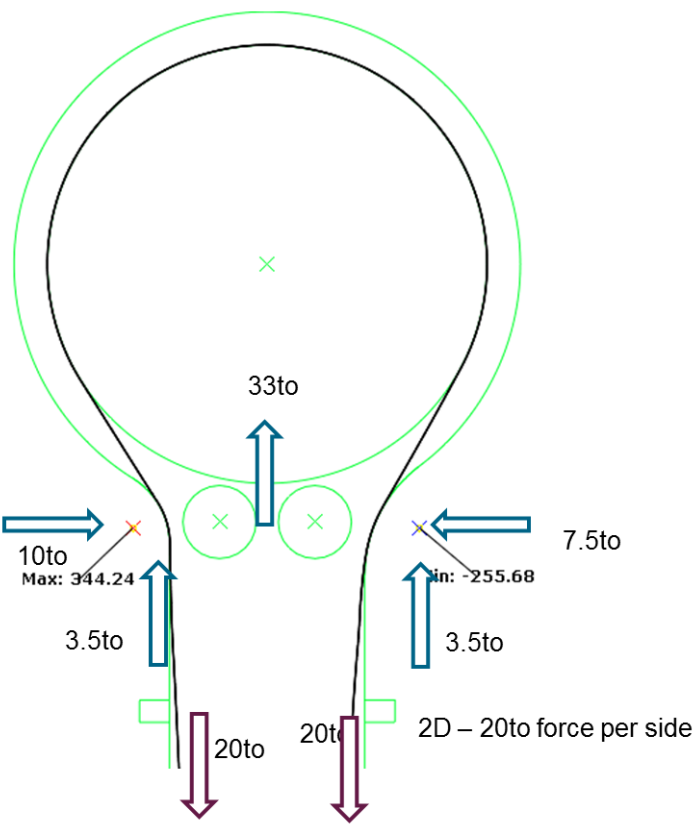

Figure 16. Loads and reaction forces

The appropriate design of the base structure was verified by simulations, and the stability of the securing strip was evaluated by finite element simulations in Abaqus/Standard (see Figures 17 and 18). Table 5 shows the admissible radius $\mathrm{R}$ to avoid plastic deformation of strip,

$$
R=(E t) /\left(2 \sigma_{\mathrm{y}}\right)
$$

where $R$ denots the admissible radius, $E$ the Young's modulus, $t$ the strip thickness and $\sigma_{y}$ the yield strength of the material. 


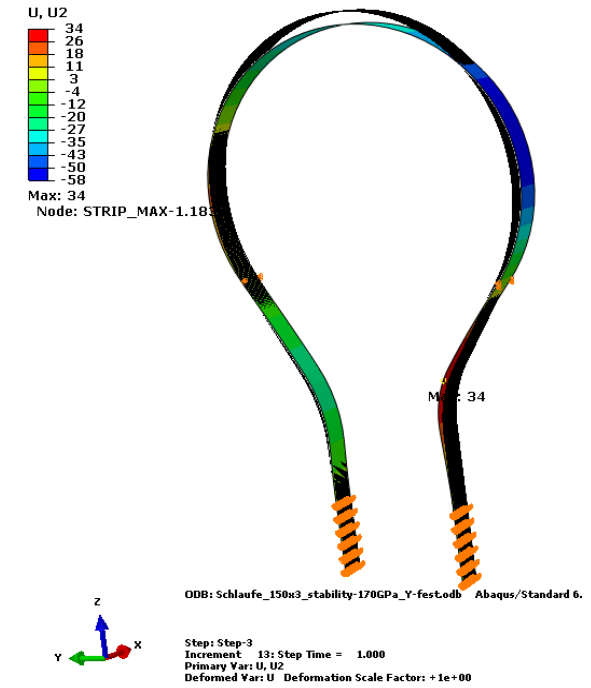

Figure 17. Deformation of the strap

As shown in Figure 17, sufficient stability is given when using a strap width of $150 \mathrm{~mm}$ and thickness of $3 \mathrm{~mm}$. The Young's modulus of hot steel with a temperature around $400^{\circ} \mathrm{C}$ is $\mathrm{E}=170 \mathrm{GPa}$.

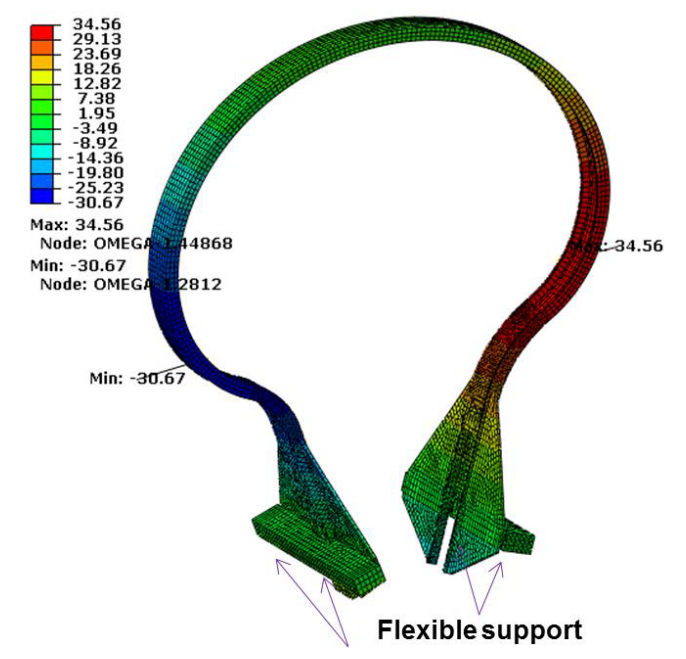

Figure 18. Deformation of the frame

Table 5. Possible dimensions for the binding strap - admissible radius $\mathrm{R}$ to avoid plastic deformation of strip

\begin{tabular}{|c|c|c|c|}
\hline $\begin{array}{c}\text { Yield strength } \\
\sigma_{y}[\mathrm{MPa}]\end{array}$ & $\mathrm{t}=2 \mathrm{~mm}$ & $\mathrm{t}=3 \mathrm{~mm}$ & $\mathrm{t}=4 \mathrm{~mm}$ \\
\hline 400 & 525 & 788 & 1050 \\
\hline 600 & 350 & 525 & 700 \\
\hline 800 & 263 & 394 & 525 \\
\hline
\end{tabular}

\section{Summary and Outlook}

For safe coil evacuation, pre-bending of the strip using a lowered roller table and a 3-roll unit can yield improvements in terms of reduced coil diameter. However, the simulations so far have not achieved total elimination of spring-back. This could be because of simulation-related issues as: (i) the hourglass-control utilised in the simulations which could yield increased stiff elements, (ii) the initial conditions with a core-coil and a rigid connection of strip to core-coil, and (ii) instabilities during the settling steps

In addition, the absolute position of the 3-roll unit and the relative position of the rolls are not optimised. There is further potential to reduce spring-back by using both measures, the lowered roller table and the 3-roll unit, in simulations.

Finally, there is scope to reduce spring-back by including the friction model described in Section 4 in the Abaqus/Explicit model for more realistic friction behaviour of the coil. Such simulations are scheduled for the near future.

The concept of the omega car provides an alternative for safe coil evacuation and transport of the coil. The requirements to this equipments would, however, be reduced when pre-bending of the strip can be achieved by the above mentioned installations in the downcoiler area.

\section{References}

1. A. Pauschitz, F. Franek, Austrian competence center for tribology AC2T; www.ac2t.at/ac2t/

2. ABAQUS/CAE V6.13-5, User's manual. Online documentation help: Dassault Systèmes (2013)

3. C. Salzmann, L. Pichler et. al., World patent, Holding together and transporting a metal band rolled up into a coil, WO2015172965A1 (2015) 